Short Report

\title{
Renin Release and Renal Pelvic Pressure in the Isolated Perfused Dog Kidney
}

\author{
Tadashi Harada, Fumikazu Sakamoto, Makoto \\ Suzuki and SEIGI Tsuchida \\ Department of Urology, Akita University School of Medicine, \\ Akita 010
}

Harada, T., Sakamoto, F., Suzuki, M. and Tsuchida, S. Renin Release and Renal Pelvic Pressure in the Isolated Perfused Dog Kidney. Tohoku J. exp. Med., 1980, 130 (2), 203-204 — The perfused dog kidney was used for a study of the relationship between renin secretion and renal pelvic pressure. The kidney was completely isolated and perfused with perfluorochemical emulsion. During the elevation of renal pelvic pressure, renin secretion rate increased from $0.93 \pm 0.43$ to $5.54 \pm 1.57 \mathrm{ng} / \mathrm{g} \mathrm{min}$, perfusion flow rate decreased temporarily and recovered within a few minutes. - _ _ renin release; renal pelvic pressure; isolated dog kidney; fluorocarbon

Considerable evidence implicates the sympathetic nervous system and the catecholamines in the regulation of renin release. Several hypotheses about renin release following ureteral constriction have been reported (Vander and Luciano 1967). Without complicating systemic effects, renin release was examined in the isolated dog kidney perfused with fluorocarbon emulsion at a constant perfusion pressure and flow rate to investigate the mechanism of renin release following pelvic pressure elevation.

Experiments were performed on isolated dog kidney. The kidney was perfused by the apparatus (pulsatile Harvard pump. Fig. 1) at the mean pressure of $122 \pm 3 \mathrm{mmHg}$ and maintained at $37^{\circ} \mathrm{C}$. The oxygenator was supplied with a mixture of $95 \%$ oxygen and $5 \%$ carbon dioxide. The perfusate was composed of modified lactated Ringer's solution containing $10 \%$ perfluorochemical emulsion (Fluosol-43, Composition: $\mathrm{Na}+130.0 \mathrm{mEq} / \mathrm{hter}$; $\mathrm{K}+4.0 \mathrm{mEq} /$ liter $; \mathrm{Ca}^{++} 2.5 \mathrm{mEq} /$ liter $; \mathrm{Cl}^{-} 112.0 \mathrm{mEq} / \mathrm{liter} ; \mathrm{HCO}_{3}{ }^{-} 12.5 \mathrm{mEq} / \mathrm{liter} ; \mathrm{Mg}^{++}$ $2.0 \mathrm{mEq} /$ liter; lactate $14.0 \mathrm{mEq} / \mathrm{liter}$; perfluorotributylamine $10.0 \mathrm{~g} / 100 \mathrm{ml}$; pluronic

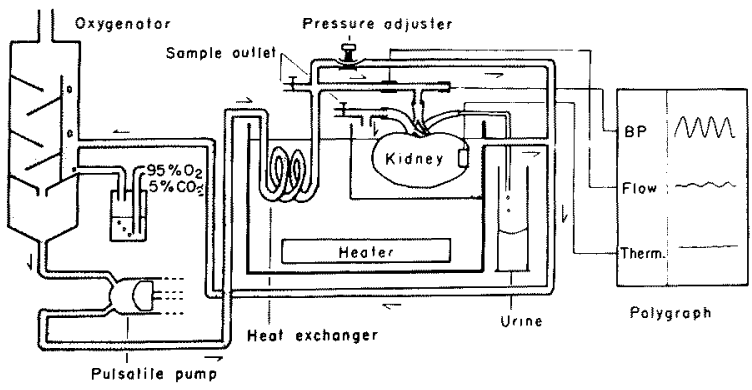

Fig. 1. Schematic diagram of perfusion system.

Received for publication, May 7, 1979. 
F $681.28 \mathrm{~g} / 100 \mathrm{ml}$; glucose $90.0 \mathrm{mg} / 100 \mathrm{ml}$; heparin sodium $2000 \mathrm{U} /$ liter ; creatinine $2.0 \mathrm{mg} /$ $100 \mathrm{ml}$; pH 7.5 7.7; osmotic pressure $290 \mathrm{mOsm} / \mathrm{liter}$. A polyethylene catheter was inserted into the ureter to collect urine. Pelvic pressure was raised to $100 \mathrm{mmHg}$ by gravity infusion through the ureteral catheter. Perfusion pressure and flow rate became stable within a few min. Experiments were conducted during steady-state perfusion. Perfusate samples for measurement of renin release were obtained immediately, at $10 \mathrm{~min}$ and at $20 \mathrm{~min}$ after the elevation of pelvic pressure, and also at $30 \mathrm{~min}$ after release of the elevation of pelvic pressure. The samples were mixed with nephrectomized dog plasma as the renin substrate. Plasma renin activity was determined by radioimmunoassay according to the modified method of Haber et al. The renin secretion rate was calculated as follows: $\mathrm{RSR}=$ (effluent $\mathrm{PRA}$-affluent $\mathrm{PRA}) \times$ perfusion flow rate, and expressed as $\mathrm{ng}$ $\mathrm{AI} / \mathrm{g}$ of kidney min.

TABLE 1. Effects of renal pelvic pressure elevation on renin release

\begin{tabular}{|c|c|c|c|c|c|c|}
\hline & \multirow{2}{*}{$\begin{array}{c}\mathbf{P f P} \\
(\mathrm{mmHg})\end{array}$} & \multirow{2}{*}{$\begin{array}{c}\mathrm{PP} \\
(\mathrm{mmHg})\end{array}$} & \multirow{2}{*}{$\begin{array}{c}\text { PFR } \\
(\mathrm{ml} / \mathrm{g} \min )\end{array}$} & \multicolumn{3}{|c|}{ Renin } \\
\hline & & & & $\mathrm{a}(\mathbf{n g}-\mathrm{AI} / \mathrm{ml})$ & $\mathrm{e}(\mathrm{ng}-\mathrm{AI} / \mathrm{ml})$ & $\begin{array}{c}\mathrm{RSR} \\
(\mathrm{ng}-\mathrm{AI} / \mathrm{g} \min )\end{array}$ \\
\hline Control & $\begin{array}{l}122.0 \\
\pm 3.0\end{array}$ & $0 \pm 0$ & $\begin{array}{l}1.80 \\
\pm 0.07\end{array}$ & $\begin{array}{l}3.56 \\
\pm 0.64\end{array}$ & $\begin{array}{l}4.12 \\
\pm 0.70\end{array}$ & $\begin{array}{l}0.93 \\
\pm 0.43\end{array}$ \\
\hline $\begin{array}{l}\text { Immediately after } \\
\text { PPE }\end{array}$ & $\begin{array}{l}128.6 \\
\pm 2.8\end{array}$ & $100 \pm 0$ & $\begin{array}{l}1.65 \\
\pm 0.08\end{array}$ & $\begin{array}{l}5.60 \\
\pm 1.26\end{array}$ & $\begin{array}{l}8.40 \\
\pm 1.27\end{array}$ & $\begin{array}{l}4.77 \\
\pm 2.29\end{array}$ \\
\hline 10 min after PPE & $\begin{array}{l}125.0 \\
\pm 2.4\end{array}$ & $100 \pm 0$ & $\begin{array}{l}1.81 \\
\pm 0.07\end{array}$ & $\begin{array}{l}2.90 \\
\pm 1.05\end{array}$ & $\begin{array}{l}5.94 \\
\pm 0.83\end{array}$ & $\begin{array}{l}5.54 \\
\pm 1.57\end{array}$ \\
\hline 20 min after PPE & $\begin{array}{l}122.0 \\
\pm 3.0\end{array}$ & $100 \pm 0$ & $\begin{array}{l}1.81 \\
\pm 0.07\end{array}$ & $\begin{array}{l}5.02 \\
\pm 0.23\end{array}$ & $\begin{array}{l}6.94 \\
\pm 0.63\end{array}$ & $\begin{array}{l}3.42 \\
\pm 1.00\end{array}$ \\
\hline $\begin{array}{l}30 \mathrm{~min} \text { after release } \\
\text { of PPE }\end{array}$ & $\begin{array}{l}120.6 \\
\pm 3.7\end{array}$ & $0 \pm 0$ & $\begin{array}{l}1.87 \\
\pm 0.09\end{array}$ & $\begin{array}{l}5.64 \\
\pm 0.59\end{array}$ & $\begin{array}{l}5.80 \\
\pm 0.39\end{array}$ & $\begin{array}{l}0.337 \\
\pm 1.21\end{array}$ \\
\hline
\end{tabular}

PfP, perfusion pressure; PP, pelvic pressure; PFR, perfusion flow rate; RSR, renin secretion rate; e, plasma renin activity of effluent; a, plasma renin activity of affluent; PPE, pelvic pressure elevation. Results are expressed as mean \pm s.E. of experiments on 5 kidneys.

Perfusion with fluorocarbon emulsion maintained constant renal function and perfusion flow rate during 3-4 hr (Noto and Harada 1979). Table 1 summarizes experiments on 5 dogs. Renal arterial pressure rose to $128.6 \pm 2.8$ (mean \pm s.. .) $\mathrm{mmHg}$, whereas the perfusion flow rate fell slightly immediately after the elevation of pelvic pressure. But, the perfusion flow rate had a tendency to recover to control level within a few $\mathrm{min}$. The elevation of pelvic pressure raised the renin secretion rate to $5.54 \pm 1.57 \mathrm{ng} \cdot \mathrm{AI} / \mathrm{g} \mathrm{min}$. Thirty min after the release of the pelvic pressure elevation, the renin secretion rate fell to control levels. These findings support a direct intrarenal effect of pelvic pressure elevation on the renin secretion rate. The increase of renin release following pelvic pressure elevation may be activated by reduced delivery of sodium to the distal nephron (macula densa mechanism), or by dilation of the afferent arterioles (autoregulation mechanism).

\section{References}

1) Noto, H.G. \& Harada, T. (1979) Viability of the isolated dog kidney perfused with fluorocarbon emulsion. Jap. J. Transplant., 14, 328-329. (Japanese)

2) Vander, A.J. \& Luciano, J.R. (1967) Neural and humoral control of renin release in salt depletion. Circulat. Res., 21, suppl. II, 69-75. 